\title{
Wargame Mapping and Implementation for Emergency Evacuation of Residents in Urban Waterlogging Disaster
}

\author{
Peng Chen ${ }^{1}$, Jiquan Zhang ${ }^{2}$, Yingyue Sun ${ }^{1}$, Xiaojing Liu ${ }^{1}$ \\ 1 School of Tourism and Geography Science of Jilin Normal University, Siping, Jilin, 136000,China. \\ pp11290@163.com, syy800201@126.com,ppsyy1129@163.com \\ 2 School of Environment of Northeast Normal University, Changchun, Jilin, 130117,China. \\ zhangjq022@nenu.edu.cn
}

Received December 31, 2017

Accepted January 15, 2018

\begin{abstract}
Urban waterlogging disaster represented a serious threat to the residents and the traffic. For the purpose of drilling, the wargame excise is introduced to the residents' emergency evacuation in urban waterlogging disaster. As an essential component of wargame, the chessboard (map) is an important tool for the study of deduction and command training. In this paper, the research focus on the methods of generating wargame map based upon vector map data, from the viewpoints of mathematic foundation as well as quantitative treatments of point, line and plane for the map composed by regular hexagonal grids. In addition, a numerical simulation model of waterlogging was built to correlate and locate the simulation results of flooded streets, emergency organizations and wargame map, so as to the comprehensive map of the emergency evacuation of residents for urban waterlogging disaster was drawn. The conclusion of the paper suggests that provide a low-cost drilling platform for emergency management agency of urban waterlogging by assessing high-risk events, evaluating and finding out shortcomings in the emergency plan.
\end{abstract}

Keywords: Waterlogging Disaster; Wargame; Wargame Map; Emergency Evacuation

\section{Introduction}

Waterlogging disaster is defined by the short heavy rain or continuous rainfall, leading to a lot of water on the road of the city, threatening the safety of residents, namely waterlogging disasters. Along with the accelerating process of urbanization, the urban waterlogging disaster occurrence frequency will be higher in the future. From the urban waterlogging disaster history statistical data shows that urban road waterlogging depth up to $1.5-3.0 \mathrm{~m}$, vehicles, pedestrians cannot normally access, underground facilities were flooded, the cottage and the basement of the inhabitant's life under serious threat. From 2008 to 2012,there are 351 cities have undergone different degrees of waterlogging, water depth of the roads in $50 \mathrm{~cm}$ above the city reached $60 \%$, water more than half an hour accounted for nearly $80 \%$, the heavy urban waterlogging disasters cities including Dalian, Beijing, Wuhan, Hangzhou, Zhengzhou, Tianjin, Harbin and so on. For example, since July 21, 2012, the most serious waterlogging disasters in Beijing city history has occurred, the affected population up to 124,000 , the city's economic losses was nearly billion, resulting in 79 people were killed; In June 18, 2012, Haihe East Road, Nangang District, Harbin,there are a serious water phenomenon due to heavy rain, including 21 large-area road water points, there are 4 serious water points, nine more serious water points, road deepest water is about $1.5 \mathrm{~m}$. In which dozens of people trapped in more than $2 \mathrm{~m}$ depth water, their lives are seriously threatened. Thus, the occurrence of urban storm waterlogging disasters have caused huge losses to urban residents ' life and property. But also to the urban construction and economic development, social stability and a tremendous negative impact, it is a serious impediment to sustainable urban development.

Wargame, called "Wargame", war games, called "Wargaming". It is a tool that use chessboard and military force to confront the pieces deduction. The wargame excise tries to deduce the future from a deeper understanding on historical data. It is also a 
"game", and even the combination of historical information and science. Playing wargame needs a chessboard, chess pieces and rules, of which the chessboard is also referred to as wargame map. The map was used in wargame will be drawn to scale in accordance with the terrain of the study area and historical contexts. The two sides (Our troops and the enemy troops) place their pieces into designated grids, where the geographic data affecting the operation (Perla,2012) will be indicated, including type of terrain, bridge, road, airport, harbor and others.

Along with the computer network information era, computer technology is widely applied to the wargame excise. Computer-assisted war games, computer wargame have become an important research means of modern information warfare (Perla, 2012) However, Wargame board production is still mainly by way of human-computer interaction of data collection, inspection and design. This method is unfavorable because of low-efficiency, error-prone, long cycle and high cost. As a result, the map digitalization becomes an important restriction in development and application of computer-aided wargame system. The map directly serves for space orientation, visibility calculation, mobile calculation, and engagement calculation of pieces. Hexagonal grids are usually used to discretize the battlefield space. Various geographic elements are described on the grids to build an environmental model. Lines as obstacle are usually modelled on the boundary of grids, such as rivers, gulley and frontiers; lines as channel are usually on the center of grids, such as roads; various planes are directly modelled on the surface of grids, such as landform elevation, vegetation, lakes, etc (Yang, 2007). Many scholars made researches on this point to build map in computer-aided wargame system. Digitalizing the wargame map by existing geographic information system software to digitalize, Yang Lun (Yu, 2010) proposed a MapX-based algorithm of hexagonal grids mapping. This approach reduces the workload of wargame to quantify terrain and improve the efficiency of drawing wargame map.Yu Xiaohua(Xu, 2009) proposed an algorithm of quick map-grids location based upon meta-heuristics. This method is described in detail map drawing, but drawing process involves more complex mathematical methods which is inconvenience to the user. $\mathrm{Xu}$ Ning(Liu, 2013) encoded the map information by run-length of grids, reducing the space greatly. All these researches were concentrated on generation, location and data storage of hexagonal grids, doing little to the environmental modelling. The maps were still generated by artificial judgment and collection, showing low efficacy. Although this method has been drawing board wargame encoding process, but the coding process is more complex, users need to master the process of geocoding in advance. Liu (Condat, 2005) made studies on algorithm of automatic map generation, in which the planes were modeled by polygon area intersection algorithm, and lines were cut into polylines over hexagonal grids. This method relied on the algorithms of polygons intersection and cutting, which was slow in generation. For this purpose, the algorithms of automatic map generation were further discussed in this paper.

In current, the most common international drawing algorithm is described in the literature (Condat, 2005), however, this approach is lack of map Localization, if binary storage lookup location algorithm is applied to draw map, and the time and space expenses of algorithm for increased dramatically, their complex degrees are o (lgn) and o (n), respectively (Thomas, 2008), it is unsatisfactory that to draw giant map in terms of time consumption. Under the concept of the relief is equivalent to war, the wargame excise is gradually applied to the disaster management (especially the rescue) and incident management system (Zhu, 2011; Simonsen, 2008). Wining the "best" profit by "game" saves cost and time, mainly used for negotiation and other competitive or confrontational affairs(Li, 2009; Darken, 2005). On the other hand, problems are detected and countermeasures are prepared earlier by "excise", mainly used for formulation and optimization of plans. Moreover, optimum and reasonable emergency management plan can be selected based upon the development of disaster, historical data and model (Li, 2012).

In this paper, the research put forward the method of mapping which is necessary for wargame excise for emergency evacuation of residents in urban waterlogging disaster, proposing the procedures, methods and visualized process of wargame mapping. It provides a map for emergency management agency to simulate the disaster happening and developing in a virtual space, and also an excise for residents, decision-makers and commanders at all levels in emergency evacuation drilling, so as to improve the emergency management level of urban disasters.

\section{Summary of Researched Area}

Ha-Erbin is the capital city of Heilongjiang province in China, it is located at $125^{\circ} 42^{\prime}-130^{\circ} 10^{\prime}$ 
east longitude and $44^{\circ} 04^{\prime}-46^{\circ} 40^{\prime}$ north latitude (as shown in Fig 1). Daoli District is a district of Harbin, which is the part of the Songnen Plain that higher in the south of the distribution than the north. Summer is hot and humid with frequent rains. The average annual rainfall precipitation is $569.1 \mathrm{~mm}$, Major precipitation occurs in June-August, which receive over $60-70 \%$ of total annual rainfall .The study area has an area over $479 \mathrm{~km}^{2}$. The total population is 0.67 million people, and downtown residents are over 0.18 million people. There are bungalows have 8334 households, the number of under the first floor households is 2185. Cottage residents and the residents of under the first floor (basement) is highly vulnerable to waterlogging disasters, they are the focus of the refuge objects.

\section{Data and Methods}

\subsection{Data Source and Processing}

Meteorological data in this paper came from Harbin Meteorological Bureau, including daily precipitation in 2010, which served as one of initial parameters for waterlogging model calculation. Basic geographic information data were derived from QuickBird image of Daoli District (resolution ratio $0.61 \mathrm{~m}$ ), DEM, land-use map, and underground drainage pipe network distribution map.

The parameters of waterlogging model is set as follows: (1) Rainfall. In August 10, 2011, Harbin began to rain. From the early morning of August 10 around 2:00 to $14: 00 \mathrm{pm}$, where local rainfall has exceeded $100 \mathrm{~mm}$. As the value of storm intensity parameter $\mathrm{q}$ in this paper; (2) Roughness coefficient value of $\mathrm{n}$. Since the roads are made of asphalt roads, so the value of $n$ is 0.012 ; (3) the elevation difference of the study area only $30.81 \mathrm{~m}$, the terrain is relatively flat, so waterlogging model has better applicability. (4) Because of road water, residents of the house will have a lot of water, especially in the basement, so cottage residents and residents of the basement are affected, a total of 10579 people. QuickBird image of researched area was interpreted by GIS technique to vectorize the information of underlying surface, and obtain the vector layers of residential areas, roads, green areas, squares, schools, open areas and emergency organizations. The interpreted underlying surface vectorgraph, DEM and other data were attribute - associated to the wargame map in the research, with every hexagonal gird corresponding to underlying geographic information, providing fundamental data for the mapping and implementation of wargame excise.

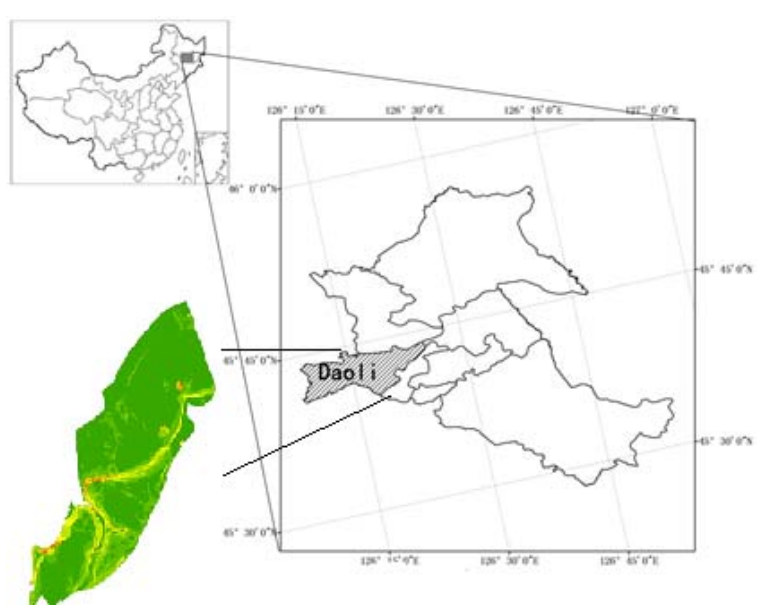

Fig 1. Diagram of the study area

\subsection{Numerical Calculation Methods of \\ Waterlogging}

The first step of wargames was draw the wargame map, second step is the initial settings of our troops and the enemy troops is constructed. Therefore, we have drawn the rescue facilities and rescue forces in the wargame map based on the actual investigation, and the enemy's initial chess layout by simulated the numerical waterlogging simulation model. Several simulation methods can be used to model waterlogging situations on urban streets and roads, but most of them only use the depth of waterlogging as the determining factor. None of the models include the velocity and direction of flowing water (Chen, 2012, 2014). In this study, the impact from both the depth and flow velocity of waterlogging was considered. Models were constructed using the two-dimensional nonsteady flow as the structure, and irregular grids were derived from the source terrain data (Zhou, 2009). In the comprehensive urban rainstorm waterlogging mathematical model, water drainage pipelines were simulated using a one-dimensional nonsteady flow method. GIS was selected as a visualization tool. The urban rainstorm waterlogging mathematical model to calculate the accumulated water of the road based on one and two-dimensional unsteady flow equations. In order to meet the wargame, the Hexagonal grid is applied to calculate the depth of road water for each grid center, regular hexagon grid edge as water exchange channels (Condat, 2005). In which the two-dimensional unsteady flow equation is used to calculate the depth of the water, One-dimensional unsteady flow equation for the calculation of water within the grid evolution, namely calculate the changes of submerged area. It will provide the basis 
information for the position of "the enemy" both pieces initial layout.

The numerical calculation methods were utilized to simulate the road water in researched area. Precipitation, the initial parameter of model, was set to $100 \mathrm{~mm}$, and the duration was $30 \mathrm{~min}$ (the practicability of numerical waterlogging model has been demonstrated in the Bibliography (Chen, 2014)). The water depth, flooding area and current speed calculated by the model will be taken as the pieces' initial attributes of "enemy" in subsequent wargame excise for emergency evacuation of residents in urban waterlogging disaster. The construction of waterlogging model has been completed, first, the mathematical basis wargame mapping were described in detail, Secondly the process of wargame mapping is carried out in detail, finally the draw wargame maps and waterlogging model mathematically combined to calculate the study area road puddles, stagnant water where the net grid is seen as "the enemy" pieces.

\subsection{Mathematic Foundation of Wargame Map}

\subsubsection{Basis for selection of map grids}

The wargame map is the battle place for the two sides, and also an important component of wargame excise for emergency evacuation of residents in waterlogging. Before the excise, the two sides place their pieces into the designated grids, similar to other board games. However, the wargame map contains geographic information like types of terrain, roads accessible or not, bridges and buildings, which has influences on the battle. This method of map with grids and terrain classification is called the "terrain quantization" or "quantitative terrain" . At present, the commonly used grids for wargame map are square and regular hexagonal. The wargame is supported by square grids is restricted by the defects of squares. In addition, since it is impossible for the troops in real battle field to act in such grids, grids allowing 360o full-directional moving are needed to reflect the actions of troop as possible, that is, the regular hexagonal grids (deviation between six sides and diagonal is in the regular hexagon; and between the four diagonals of square is ). The deviation of grids is the minimum in regular hexagonal grids $(\mathrm{Li}$, 2014)(Fig2-Fig3). However, such wargame map is only applicable for military operations, but not for the research. In this paper, a wargame map with regular hexagonal grids was built and associated to underlying information. Every side of the hexagon was considered as the water exchange channel between grids, and the water level was calculated on the center of form. A numerical simulation model of waterlogging was built based upon the regular hexagonal grids. At this moment, the wargame map contains waterlogging information, with flooded grids as "enemy" pieces. It provides a basic map of wargame excise for emergency evacuation of residents in urban waterlogging disaster.
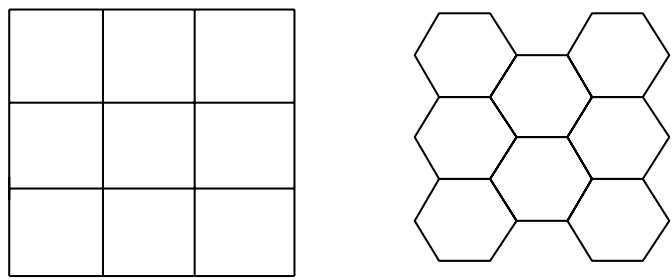

Fig 2. Square and Regular Hexagonal Grids
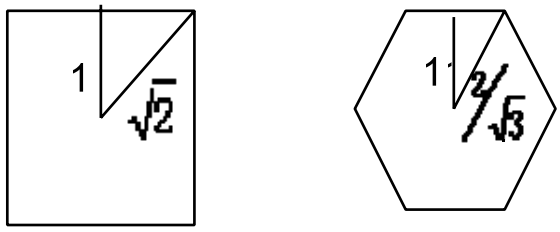

Fig 3. Comparison between Deviations of Square and Regular Hexagonal Grids

\subsubsection{Method of mapping regular hexagonal grids}

(1) Calculate the center point of regular hexagonal grids

The method of mapping regular hexagonal grids is divided into two steps. Firstly, the coordinates of center points are solved for the grids. Then, the coordinates of six top points are calculated from the relative position of center and top points. Let $r$ be the side length of regular hexagon, $i$ and $j$ are row and column numbers of grid, respectively. The formula for coordinates of center point is:

$$
\left\{\begin{array}{l}
x_{i}=\frac{3}{2} r \times i+\frac{1}{2} r \\
y_{i}=\left\{\begin{array}{lr}
\sqrt{3} r \times j+\frac{\sqrt{3}}{2} \times r, i \%=0 \\
\sqrt{3} r \times j, i
\end{array} \quad \% 2=1 .\right.
\end{array}\right.
$$

where, $\%$ is remainder operation.

(2) Convert the codes of regular hexagonal grids into rectangular coordinate system

There are three coding modes currently: recording the row and column numbers of regular hexagonal grids in non-regular rectangular coordinate system; coding the regular hexagonal grids in oblique coordinate system; and coding the regular hexagonal grids in symmetric axis 
coordinate system. The three modes have their advantages and disadvantages. By comparing the conversion efficiency, storage efficiency of grids and neighbor searching speed among the three, the researcher finally decided to adopt the first mode (Fig4). The coordinates of center point can be calculated by formula (7).

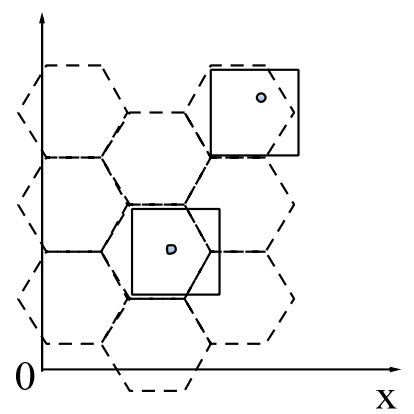

Fig 4. Rectangular Coordinate System

(3) Convert the rectangular coordinates into codes of regular hexagonal grids

Let the coordinate system of a point $(x, y)$. The point is converted to local coordinate system by formula (2), as shown in Fig 5.

$$
\left\{\begin{array}{l}
i^{\prime}=\operatorname{int}\left(\frac{x}{\text { width }}\right) ; \\
j^{\prime}=\operatorname{int}\left(\frac{y+\frac{i^{\prime} \% 2}{2} \text { heigth }}{\text { height }}\right) ; \\
x^{\prime}=x-\text { width } \times i^{\prime} ; \\
y^{\prime}=y-\text { height } \times j^{\prime} .
\end{array}\right.
$$

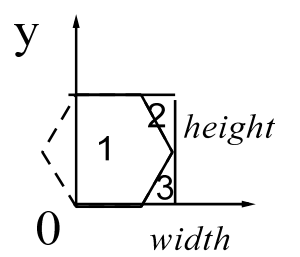

Fig 5. Local Coordinate System

In the local coordinate system, it is necessary to determine the region of grids where the point locates. Therefore, the following operations will be taken to determine the region of point and code of grids.

Let $d y=y^{\prime}-$ width, $d x=x^{\prime}-\frac{1}{2} h e i g h t, i$ and $j$ are codes of regular hexagonal grids where the point locates.

1) If $d y<\frac{\sqrt{3}}{2} d x$ (point in region 3), then

$$
\left\{\begin{array}{l}
i=i^{\prime}+1 ; \\
j= \begin{cases}j^{\prime}-1, i^{\prime} \% 2=1 \\
j^{\prime}, \quad i^{\prime} \% 2=0 .\end{cases}
\end{array}\right.
$$

2) If $d y<-\frac{\sqrt{3}}{2} d x$ (point in region 2), then

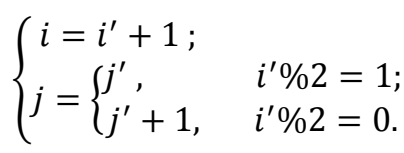

3 ) If the point is in region 1 , then

$$
\left\{\begin{array}{l}
i=i^{\prime} \\
j=j^{\prime}
\end{array}\right.
$$

\section{Wargame Mapping for Waterlogging Disaster}

\subsection{Mapping Procedures}

(1) Select the projection

Wargame map is the carrier for describing the fight in real battle field and the excise of commander on two-dimensional plane. Drawing on two-dimensional plane will certainly cause the deformation of regions on the map. Therefore, the projection with minor deformation should be selected in large-scale map. Gauss projection satisfies this requirement by its isogonism and minor area deformation in small regions. Therefore, Gauss projection was selected in this paper as the wargame map projection for emergency evacuation of residents in urban waterlogging disaster.

(2) Determine the scope of researched area

After the projection is selected, the scope of researched area should be determined for the wargame mapping. The scope of researched area is determined by projection coordinates of the four top points, that is, the horizontal and vertical minimums are the lower left corner, and horizontal and vertical maximums are the upper right corner of the map.

(3) Determine the size of regular hexagonal grids

The size of regular hexagonal grids is the criterion for deciding the accuracy of wargame deduction results for emergency evacuation of residents in urban waterlogging disaster. It should be determined based upon the level, type and rule of deduction as well as the operation efficiency of computer. Too small grids division leads to lower calculation of computer. Usually, the size of grids for wargame map is $50-200 \mathrm{~m}$ (Colin, 2007). 
(4) Determine the origin of coordinate

The origin of coordinate represents the type of coordinate system. Since the wargame map is deduction on two-dimensional plane, the lower left corner is taken as the origin of coordinate. The division is shown as Fig 6.

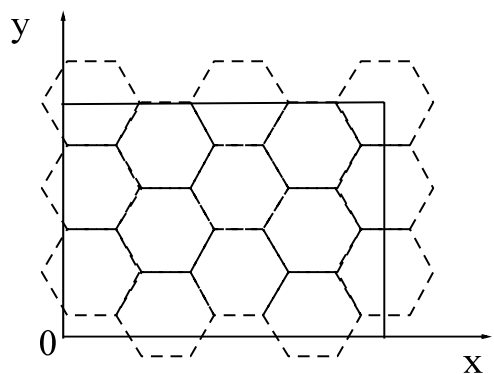

Fig 6. Hexagonal Grids of Wargame Map

\subsection{Algorithm of Wargame Map}

The wargame map was drawn with GIS technique is vector data that characterized by matched attributes and primitives. By attribute association of GIS, the wargame map is given the attribute information of underlying surface, offering basis for the operations of two sides. Common GIS components like MAPX, ArcEngine can be used in combination with advanced programming language platform to program and draw the map. The principles of mapping algorithm are as follows.

At first, a horizontal primitive is created, and then drawn cyclically on the map [3], as follows:

A regular hexagonal grid is considered as a hexagon composed by six line segments in the same length with $120^{\circ}$ included angle. The side length is a, the primitive is drawn as Figure 6 , in which segments , $P_{11} P_{12}, P_{12} P_{13}, P_{13} P_{14}$ and $P_{14} P_{15}$, have equal length a, and the included angle is $120^{\circ}$. In the coordinate system on the screen, if $P_{11}$ $\left(x_{11}, y_{11}\right)$ is known, the coordinates of $P_{12}$ $\left(x_{12}, y_{12}\right), P_{13}\left(x_{13}, y_{13}\right) 、 P_{14}\left(x_{14}, y_{14}\right)$ and $P_{15}\left(x_{15}, y_{15}\right)$ can be calculated, where:

$$
\begin{gathered}
x_{12}=x_{11}+a, y_{12}=y_{11} \\
x_{13}=x_{11}+1.5 a, \quad y_{13}=y_{11}+\sqrt{3} / 2 a \\
x_{14}=x_{11}+2.5 a, y_{14}=y_{11}+\sqrt{3} / 2 a \\
x_{15}=x_{11}+3 a, \quad y_{15}=y_{11}
\end{gathered}
$$

(2) After the scope of researched area is determined, the primitive 1 is cyclically drawn on axis $\mathrm{x}$ and $\mathrm{y}$ to obtain the regular hexagonal grids of researched area. The width of grids is Width, and height is Height, with $\mathrm{C}++$ code as:

$$
\begin{aligned}
& \text { for }(\mathrm{i}=0 ; \text { Height } / ; \mathrm{i}++) \\
& \left\{\begin{array}{c}
\text { for }(\mathrm{j}=0 ; \text { Width } / ; j++) \\
\text { DrawGraphi }\left(\mathrm{i}^{*} 3 \mathrm{a}+\mathrm{j}^{*}\right) ;
\end{array}\right. \\
& /{ }^{*} \text { Drawing Primitive } / \\
& \}
\end{aligned}
$$

By calculations as above, the result of regular hexagonal grids mapping is shown as Figure 7. Superposition algorithm operates on the drawn grips with basic vectors of researched area, giving the grids information of underlying surface. These grids are also basic skeleton for numerical calculation of urban waterlogging. In combination with the numerical waterlogging model as described in 2.2, the flood depth and scope in the grids are calculated (Fig 8).

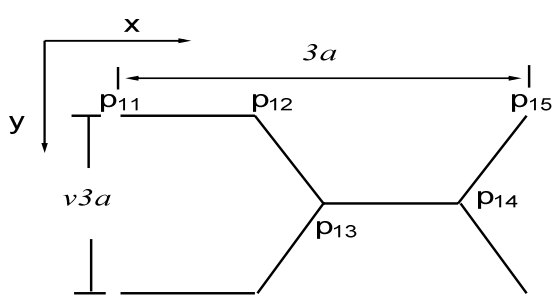

Fig 7. Primitives Creation (Screen Coordinates)

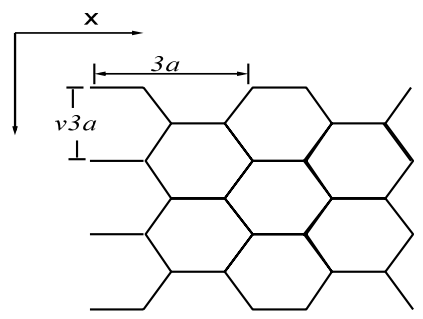

Fig 8. Result of Regular Hexagonal Grids

\section{Elements Quantization of Wargame for \\ Emergency Evacuation of Residents in Urban Waterlogging Disaster.}

\subsection{Quantization of Points}

In the wargame, the point data include residential areas, flooded areas, emergency organizations, etc. The vectorization of point elements lays foundation for the subsequent positioning of pieces, with residential areas and emergency organizations as "our" pieces, and flooded areas as "enemy" pieces. At the beginning of deduction, the residential areas and emergency organizations are placed into proper grids as per their real locations, with basic attributes preset in initial state, such as searching and rescuing capacity of emergency organizations, age, height 
and sex of residents, etc. By this way, "our" point elements are quantized to wargame map.

The pieces of "enemy" are quantized by the numerical calculation of waterlogging as described in 3.2. The flooding states of regular hexagonal grids are calculated, and these grids are considered as pieces of "enemy". The flood depth, current speed and other data calculated will be given to the grids as initial attributes of pieces.

\subsection{Quantization of Lines}

In the wargame excise, line elements involved in residents' evacuation and sheltering are mainly urban roads. The roads on the map are generally quantized by the connection between center points of two hexagonal grids. The process of roads modelling is essentially the process of vector line gridding, which is different from conventional gridding. The grids are regular hexagonal, but not square. Therefore, the process of gridding is rather complicated. The process of vector line gridding covers full route, eight direction and constant density methods(COLIN P D,2007). The eight-directional gridding is characterized by the finest grids without lines "adhesion", keeping eight directions connected. The full-route gridding method was selected in this paper. It is most convenient for detecting the influence of grids or determining the grids where the vector lines locate.

A road is considered to be composed by many line segments end to end. The roads are quantized segment by segment until all line segments are covered. Figure 9 shows the quantization of roads. In the figure, the ends of a line segment distribute in grids in two cases: starting and ending points at the same grid; starting and ending points at different grids. For the former case, formula (6) (8) can be used to determine the grids where the line segment locate, and for the latter, the grids where the connection line between starting and ending points locate should be recorded. In such case, it can be quantized by full-route gridding, as described in the following. (1) Lines parallel to $\mathrm{x}$-axis are drawn along the $\mathrm{y}$-axis direction and coded as $1,2, \ldots, \mathrm{n}$. These lines divide the xoy plane into several band regions. (2) The regions where the starting and ending points of line segment locate are determined, as well as lines intersecting the line segment, as shown in Fig 9. The lines intersecting the line segment $\mathrm{L}$ are $j_{1}, j_{2}$ and $j_{3}$. (3) The intersection points between lines and line segment are calculated, and the codes of grids where the intersection points are determined. (4) The grids where the neighboring intersection points go through are calculated.

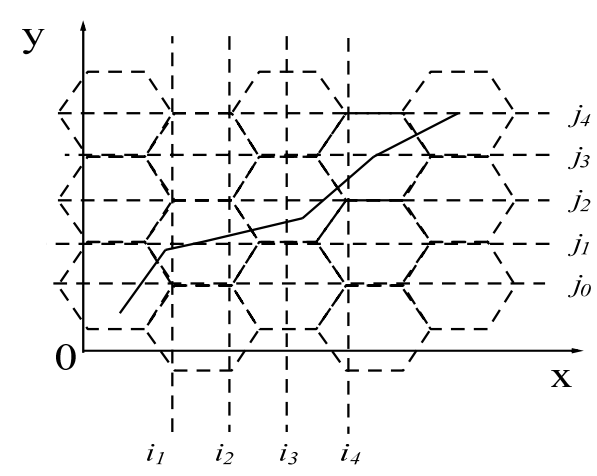

Fig 9. Quantization of Roads

\subsection{Quantization of Planes}

The plane elements are usually quantized by the method of dominant area. The simplest is to judge the intersection area of every hexagonal grid with plane. However, when the plane is large, the intersection areas of all grids in the coverage will be calculated to obtain the final result, wasting a lot of time. Therefore, only the boundary of plane needs to be found, and then all plane elements are filled.

According to this kind of thought, the algorithm of plane quantization was improved in this paper, as described in the following.

(1) Boundary tracking. The method for road quantization was used to record the hexagonal girds where the boundary lines of planes pass through.

(2) Areas judgment. Intersection area between the passing-through grids and plane elements were calculated and compared with the area of grids in accordance with the requirements.

(3) Hexagonal grids filling. According to the boundary, all hexagonal grids were identified. In the hexagonal grids system, the area filing algorithms for polygon are boundary filling and scan line filling. The boundary filling is simple, but it belongs to depth recursion consuming a lot of time. The scan line filling was adopted in this paper to identify the polygons in the boundary, as shown in Figure 10. Scan lines parallel to $y$-axis are drawn over the $\mathrm{x}$-axis. The intersection area between scan lines and polygons are calculated, and all the polygons in the area should be identified.

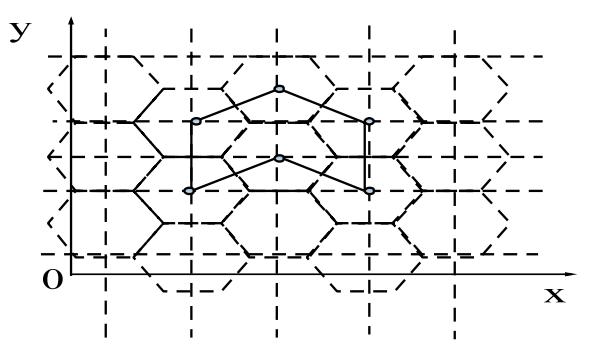

Fig 10. Planes Filling Algorithm 


\subsection{Application Example}

The wargame map is a basic component of deduction for residents' emergency evacuation process in waterlogging disaster. It is the battle place for the two sides. The wargame map is realized on computer, solving the difficulties and making up the accuracy deficiency of conventional hand drawing. Moreover, the vectorized map drawn by computer can generate grids in different scales as necessary, where the surface features and pieces can be located. In this paper, on the basis of mapping and elements quantization methods as above, the wargame map for emergency evacuation of residents in urban waterlogging disaster was programmed and drawn on ArcEnging10 and Microsoft Visual Studio 2010 platforms. In the map, the side length of hexagonal grids is $30 \mathrm{~m}$ (to be adjusted as necessary), and the area is about $2.3 \mathrm{~km}^{2}$. To make the display of wargame map clearer, the quantized roads layer and plane buildings layer were topologically associated with the grids layer in this paper to endow the grids with information of roads and buildings. The roads information in the grids was identified by " 1 ", and planes were " 2 ". In the excise, the pieces would not move along the grids identified by " 2 ", but along the direction of " 1 ". That is the rule for moving of residents and emergency organizations.

In the initial state, only pieces of emergency organizations, emergency shelters and flooded areas were placed on appropriate positions of map. Emergency organizations, emergency shelters were located as per their actual geographical positions, and the flooded areas were located relying on the result of numerical simulation waterlogging in this paper. The pieces were placed into corresponding grids (Fig11), which including emergency units, emergency refuges and water position.

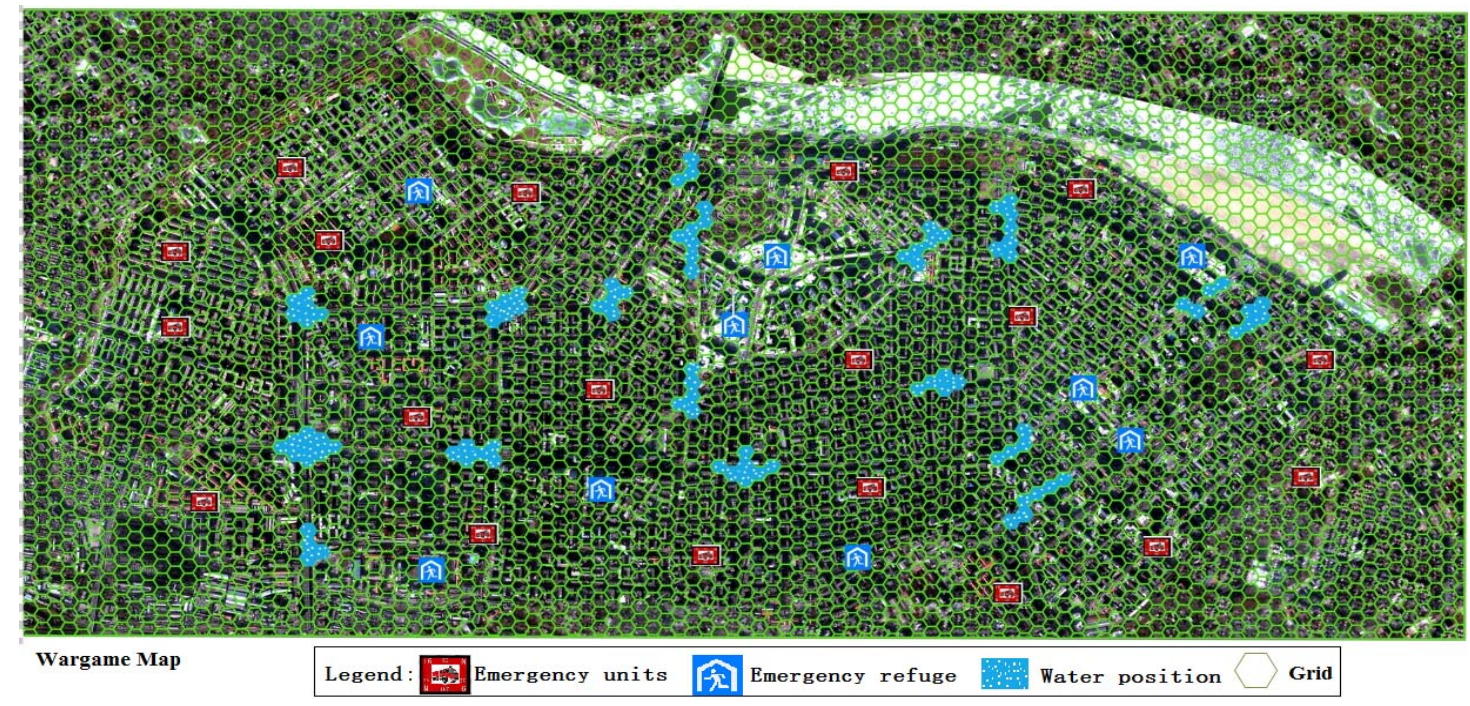

Fig 11. Wargame Map for Emergency Evacuation of Residents in Urban Waterlogging Disaster

\section{Conclusion}

In this paper, Daoli District of Harbin was taken as example to analyze the characteristics of wargame map with regular hexagonal grids. On this basis, the terrain quantization and geographic information components were combined in wargame simulation system to digitize the map making use of existing geographic information software. The procedures, methods and visualized process of wargame mapping were proposed in this paper. Moreover, based upon the numerical waterlogging model, the flooded streets, emergency organizations and shelters were located on the map as pieces in initial state, realizing the wargame map for emergency evacuation of residents in urban waterlogging disaster, and laying foundation for the subsequent wargame excises. This method fully reflects the properties of geographic information system component. It is simple and practicable, reducing the workload of terrain quantization, providing basic service platform of implementing wargame excise, and accordingly providing basis of decision-making for deduction and drilling of residents' emergency evacuation in waterlogging disaster.

\section{Acknowledgments}

Fund project: the national natural science foundation of China (41501557, 41501559, 41371495); Jilin province department of youth fund (20150520081JH). 


\section{References}

Perla P. The Art of Wargaming. U.S: Naval Institute Press, 2012.

Yang Z N. Wargame: War Game Simulation. Beijing: People's Liberation Army Publishing House,2007,1. (In Chinese)

Yang L, Peng C G, Huang J, Huang K D. Research and implementation of terrain quantization algorithm in wargame system. Computer Simulation Comput Simul, 2008,25(9):97-99. (In Chinese)

Yu X T, Li D H, Pan Y. Research and implementation of fast digital algorithm for wargame map. Computer \& Digital Engineering,2010,38(11):52-54. (In Chinese)

$\mathrm{Xu}$ N. Research and Implementation of Key Techniques of Computer Wargame. Shenyang: Northeastern University, 2009. (In Chinese)

Liu Y, Qiao X,Yu J H. Methods and implementation for terrain quantization of qargame map. Command Information System and Technology,2013,4(3):71-75. (In Chinese)

Condat L, Van De Ville, Blu T. Hexagonal versus orthogonal lattices: a new comparison using approximation theory, Proceedings of the 2005 IEEE International Conference on Image Processing (September 11-14, 2005, Genova, Italy), Vol. III, pp. 1116-1119.

Thomas H C, Charles E L, Ronald L R, Clifford S. Introduction to Algorithms (Second Edition). Cambridge MA, US: The MIT Press, 2001.

Zhu W, Chen C K, Ji D X, Sun Y F. Analysis on the risk evolution process of rainstorm disaster in cities of North China. Journal of Catastrophology, 2011, 26(3): 88-91. (In Chinese)

Simonsen I, Buzna L, Petersk, et al. Transient dynamics increasing network vulnerability to cascading failures. PhysicalReviewLetters,2008,100(21):1-4.

$\mathrm{Li} J$, Ji Z. The design and implementation of "AKY-MCP" a mobile major accidents monitoring and command platform. Journal of Safety Science and Technology, 2009, 5(6):77-80. (In Chinese)

Darken R, McDowell P, Johnson E. Projects in VR: the Delta3D open source game engine. IEEE Computer Graphics and Applications, 2006, 25(3): 10-12.

Li Y L, Yao F. Confrontational CISR simulation training system based on high level architecture. Command Information System and Technology, 2012,3(5):5-7. (In Chinese)

Chen P, Zhang J Q, Jiang X Y, Liu X P, BaoY L, Sun Y $Y$. Scenario simulation-based assessment of trip difficulty for urban residents under rainstorm waterlogging. International Journal of Environmental Research and Public Health. 2012, 9(6): 2057-2074.

Chen P, Zhang J Q, Zhang L F, Sun Y Y. Evaluation of resident evacuations in urban rainstorm waterlogging disasters based on scenario simulation: Daoli District (Harbin, China) as an example. International Journal of Environmental Research and Public Health. 2014, 11(10): 9965-9978. (In Chinese)

Zhou C H, Ou Y, Ma T. Progresses of geographical grid systems researches. Prog. Geogr. 2009,28,657-662.

Li K W, Wu Z Q, Zhang X, Li W J. Research and implementation on generataion of wargame map.
Journal of Geomatics Science and Technology, 2014, 31(4): 436-440.

Colin P D. rectangular and hexagonal grids used for observation, experiment and simulation in ecology. Ecological Modeling, 2007, 5(3):347-359. 